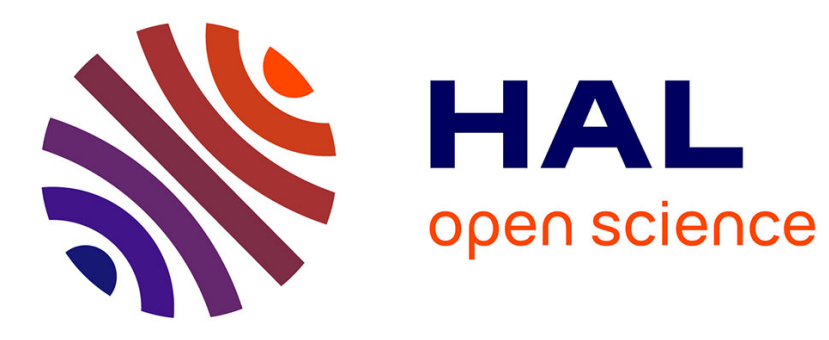

\title{
Supervised Classification with Short-Term Memory of Sleep Stages Using Cardio-Respiratory and Body Movement Variables
}

Asma Gasmi, Vincent Augusto, Paul-Antoine Baudet, Jenny Faucheu, Claire Morin, Xavier Serpaggi

\section{To cite this version:}

Asma Gasmi, Vincent Augusto, Paul-Antoine Baudet, Jenny Faucheu, Claire Morin, et al.. Supervised Classification with Short-Term Memory of Sleep Stages Using Cardio-Respiratory and Body Movement Variables. 17th International Conference on Automation Science and Engineering (CASE 2021), Aug 2021, Lyon, France. emse-03278598

\section{HAL Id: emse-03278598 \\ https://hal-emse.ccsd.cnrs.fr/emse-03278598}

Submitted on 8 Nov 2021

HAL is a multi-disciplinary open access archive for the deposit and dissemination of scientific research documents, whether they are published or not. The documents may come from teaching and research institutions in France or abroad, or from public or private research centers.
L'archive ouverte pluridisciplinaire HAL, est destinée au dépôt et à la diffusion de documents scientifiques de niveau recherche, publiés ou non, émanant des établissements d'enseignement et de recherche français ou étrangers, des laboratoires publics ou privés. 


\title{
Supervised Classification with Short-Term Memory of Sleep Stages using Cardio-respiratory and Body Movement Variables
}

\author{
Asma GASMI1,2, Vincent AUGUSTO1 ${ }^{1}$, Paul-Antoine BEAUDET2 ${ }^{2}$, \\ Jenny FAUCHEU ${ }^{3}$, Claire MORIN ${ }^{4}$, Xavier SERPAGGI ${ }^{5}$ and Franck VASSEL ${ }^{2}$
}

\begin{abstract}
In the context of the Internet of Things (IOT) healthcare, biophysical features collected during sleep needs robust analysis methods to be efficiently used to detect sleep disorders. In this paper, analysis methods using a limited number of input variables (cardiac, respiratory, and body movement) have been used to perform the classification of sleep stages. The efficiency of each classification method has been compared to a reference method that combines a large number of biophysical features referred to as PolySomnoGraphy (PSG). Five classical machine learning methods were evaluated by testing their accuracy on the same collected data. Finally, using a neural network with a short memory method, the classification task fitted $91.34 \%$ of the PSG classification.

Sleep stages, machine learning, supervised classification, sleep architecture, polysomnography
\end{abstract}

\section{INTRODUCTION}

The monitoring of sleep quality has attracted the attention of researchers for decades and more recently the use of smart technology in healthcare has been advancing steadily. In this context, the Internet of Things (IoT) technology enables to monitor sleep quality using smart wearable devices and smart furniture, without uncomfortable medical setups plugged onto the patients such as PolySomnoGraphy (PSG) considered as a reference method for sleep analysis. PSG is based on quantitative sleep parameters determined using recordings of various bio-physical features through the night (brain waves, heart rate and breathing, oxygen level in blood, eye and leg movement). Such recording setup consists in multiple sensors attached to the body of the patient such as electroencephalography (EEG) sensors, pulse oxymetry sensors, electrocardiography (ECG) sensors, ballistocardiography (BCG) sensors, nasal and oral airflow sensors, electrooculography (EOG) sensors and electromyography (EMG) sensors.

While PSG has demonstrated its efficiency for sleep analysis, the setup is expensive and uncomfortable for the patient. The recordings are usually performed for few nights in a row. Thus PSG is suitable to detect regular sleep Corresponding author:

Asma GASMI

disorders, but it is not suitable for longitudinal studies over several weeks that can be efficient to detect cognitive frailty. Therefore, minimally invasive smart devices for sleep quality monitoring can have great societal impact at low expense rate. The question addressed in this paper focuses on how to maximise the sleep stage classification accuracy while minimising the number of biophysical features analysed. In the following, we consider three stages:

1) Wake stage, characterised by slow muscle activity and slow eye movement. It represents less than $2 \%$ of the total sleep duration in standard sleep conditions.

\footnotetext{
1 Mines Saint-Etienne, Univ Clermont Auvergne, CNRS, UMR 6158 LIMOS, Center for Biomedical and Healthcare Engineering, F-42023 SaintEtienne France name.surname@emse.fr

${ }^{2}$ ATELIERS DU HAUT FOREZ, ZAC La Gravoux, 42380 LA

TOURETTE France

${ }^{3}$ Mines Saint-Etienne, CNRS, UMR 5307 Laboratoire Georges Friedel,

Centre SMS, F-42023 Saint-Etienne France

${ }^{4}$ Mines Saint-Etienne, Univ Lyon, Univ Jean Monnet, INSERM, UMR 1059 Sainbiose, Centre CIS, F-42023 Saint-Etienne France

${ }^{5}$ Mines Saint-Etienne, Institut Henri Fayol, D' epartement ISI, F-42023' Saint-Etienne France'
} 
2) Non-Rapid Eye Movement (NREM) stage, characterised by a decreased heart rate, an absence of eye movement, muscle relaxation, and a slow breathing, as well as specific brain activities. It represents $75-80 \%$ of the total sleep duration in standard sleep conditions;

3) Rapid Eye Movement (REM) stage, characterised by an intense brain activity, an absence of muscle activity, rapid and irregular breathing, increased heart and breathing rates, and quick eye movements. It represents $20-25 \%$ of the total sleep duration in standard sleep conditions.

In literature, research works present analysis methods for sleep stage classifications using partial PSG features. The accuracy of such methods is calculated compared to the full PSG method. For instance, accuracy from $74.10 \%$ to $92.04 \%$ are reached using only EEG features [31], [32], [33], [20], [26]. A 92.04\% accuracy is obtained by Nakamura et al. [34] in a study comprising 22 subjects using Support Vector Machine method. They have used Supervised method based on features calculated from spectral domain of the EEG signals. Other studies consider only the ECG features and obtain an accuracy ranging from $76 \%$ to $89.2 \%$, or only BCG features and obtain an accuracy around 75\% [17], [22], [24], [25], [14], [15]. EOG and EMG features were also considered with similar accuracy results. Combination of different bio-signals can lead to a better sleep stage classification. For example Fonseca et al. [29] succeeded in classifying 3 sleep stages with $80 \%$ accuracy. They performed a Linear Discriminant Analysis (LDA) to assess 142 variables extracted from ECG features and thoracic respiratory effort using plethysmography. Finally, these previous works highlights that even partial PSG can provide consistent data for sleep stage classification.

In this study, we focus on biophysical features that can be obtained using cost-effective and contactless sensors such as cardiac, respiratory and movement sensors. Indeed, such sensors based on accelerometers are already integrated in wearable and smart devices. This study highlights that a sleep quality monitoring system using only cost-effective sensors can provide accurate classification of sleep stage if an adequate and robust method is performed for data analysis.

The goal of this article is to develop an automatic method of sleep stage classification using simple data collected in a non intrusive way. This method must have at least $85 \%$ precision and must use the minimum number of features possible while guaranteeing the detection of all the stages correctly.

The scientific contribution lies in a new supervised classification method in association with variables that can be obtained in a non-invasive way through the use of a medical bed that collects cardio-respiratory variables (that could be derived from BCG) and tracks the body movements of the user.

This article is organised as follows. In Section II the definitions and the position of the studied problem are described. We present in Section III the methods based on the use of cardio-respiratory variables and on the combination of cardio-respiratory and movements variables. In section IV, some results are given and discussed. Section $\mathrm{V}$ gives some conclusions and perspectives for future works.

\section{POSITION OF THE PROBLEM}

This research work is made within the smart bed project. This project aims to have a bed that help supervise the sleep of the elderly living in resting homes. The supervision of the sleep must be done in a contactless way to not disturb the patient. So as a first step in defining our problem, we have started by looking at the bio-signals that could be detected in a non invasive way such as the cardio-respiratory variables and the body movement.

Every sleep stage has its own characteristics that could be observed as the variation of the vital signals. For example, variability of the heart signals can reveal useful information from the nerves system and help pathology detection. Hence many researchers have based their classification method on variation of the heart rate (RR interval, which is the duration between two R-peaks). Various cardio-respiratory features can be derived from the RR interval. Table I presents the list of useful variables for our problem.

From these characteristics, we formulate our problem as a supervised classification problem for sleep stages using data collected during sleep. In the following, we formally define the aforementioned classification problem and necessary features. 
Definition 1 (Epoch and night): An epoch $e$ is a period of time that has a short duration compared to the whole night (e.g. 30 seconds). A night $N$ is defined as a set of $n \in N$ epochs such as $n=\left\{e_{1}, e_{2}, \ldots, e_{n}\right\}$.

Definition 2 (Feature space): Let $F=\left\{f_{1}, \ldots, f_{n}\right\}, n \in \mathrm{N}$ be the feature space. $F$ represents the collected signals from the different sensors that characterise each epoch of the night.

TABLE I

VARIABLES DEFINITION

\begin{tabular}{|l|l|}
\hline Feature & Definition \\
\hline RR Interval & RR interval for every second \\
\hline $\begin{array}{l}\text { Mean RR } \\
\text { Interval }\end{array}$ & $\begin{array}{l}\text { Mean value of RR interval for the } \\
\text { whole night }\end{array}$ \\
\hline HBM & $\begin{array}{l}\text { Heartbeat mean value for every } \\
\text { 30s }\end{array}$ \\
\hline Mean HB & $\begin{array}{l}\text { Heartbeat mean value for the } \\
\text { whole night }\end{array}$ \\
\hline HRV & Heart rate variability for every 30s \\
\hline Mean HRV & $\begin{array}{l}\text { The mean value of the heart rate } \\
\text { variability for the whole night }\end{array}$ \\
\hline R & R-algorithm given in [13] \\
\hline RR & $\begin{array}{l}\text { Respiration rate } \\
\text { Respiration rate mean value for } \\
\text { the whole night }\end{array}$ \\
\hline Mean RR & Respiration rate variation \\
\hline RRst & $\begin{array}{l}\text { Respiration rate variation mean } \\
\text { value for the whole night }\end{array}$ \\
\hline Mean RRst & $\begin{array}{l}\text { Boolean indicating leg movements } \\
\text { detected through the difference of } \\
\text { the applied force on the sensors }\end{array}$ \\
\hline Move
\end{tabular}

Example 1: Table II presents an example of feature space. Each line describes one 30-second epoch of 1 patient. Line of patient 1 , epoch 1 is modelled as $\{76,425, \ldots, 1\}$.

TABLE II

FEATURE SPACE EXAMPLE

\begin{tabular}{|l|l|llll|}
\hline $\begin{array}{l}\text { Patient } \\
\text { id }\end{array}$ & $\begin{array}{l}\text { Epoch } \\
\text { id }\end{array}$ & $\begin{array}{l}\text { Heart } \\
\text { rate }\end{array}$ & RR int. & $\ldots$ & $\begin{array}{l}\text { Move } \\
(0 / 1)\end{array}$ \\
\hline 1 & 1 & 76 & 425 & $\ldots$ & 1 \\
1 & 2 & 76 & 425 & $\ldots$ & 0 \\
$\ldots$ & $\ldots$ & $\ldots$ & $\ldots$ & $\ldots$ & $\ldots$ \\
\hline
\end{tabular}

Definition 3 (Target set): Let $T=0,1,2$ be the Target set where 0 is Wake, 1 models a NREM stage and 2 models a REM stage.

Definition 4 (Sleep stage classifier): Each epoch is associated to a set of features and each epochs belongs to a certain sleep stage. Then an epoch $e$ is related to a couple $(x, y)$ where $x$ is a vector of features that occurs during epoch $e$ and $y$ is the sleep stage of epoch $e$. Let $f$ be a sleep stage classifier such as:

$$
\forall i \in[1, n], f: x_{i} 7 \rightarrow f\left(x_{i}\right)=y_{i} \in\{0,1,2\}
$$

In this article, we propose an original method to classify each epoch from a recorded night, based on the features collected for each epoch. In the following section, we present three classification methods to solve this problem. 


\section{CLASSIFICATION METHODS}

\section{A. Empirical approach}

The empirical method to detect sleep stages is based on the algorithm developed by Kurihara and Watanabe [13]. Using several features (including RR interval), we establish a decision process based on conditions to set the thresholds to classify each epoch sleep stages, as described in Table III. Coefficients $a_{1}, \ldots, a_{5}$ are used to optimize the method depending on user's characteristics and are determined using a design of experiments.

Figure 1 describes the procedure using the conditions from Table III. Such empirical method is highly dependant on the values of coefficients $a_{i}$. We performed a design of

TABLE III

CONDITIONS FOR CLASSIFIERS [14]

\begin{tabular}{|l|l|}
\hline$C_{1}$ & $\begin{array}{l}\text { Heartbeat mean value for every epoch } \\
(\text { epoch }=30 \mathrm{~s})<a_{1} \times \text { Heartbeat mean value for } \\
\text { the whole night }\end{array}$ \\
\hline$C_{2}$ & R-algorithm given in $[13] \in\left[I_{R E M}\right]$ \\
\hline$C_{3}$ & Respiration rate $<$ Respiration mean rate \\
\hline$C_{4}$ & $\begin{array}{l}\text { Heart rate variability } \leq a_{2} \times \text { The mean value } \\
\text { for the whole night of heart rate variability }\end{array}$ \\
\hline$C_{5}$ & $\begin{array}{l}\text { Respiration rate variation } \leq a_{3} \times \text { Respiration } \\
\text { rate variation mean for the whole night }\end{array}$ \\
\hline$C_{6}$ & $\begin{array}{l}\text { Heartbeat mean value for every epoch } \\
(\text { epoch }=30 s)<a_{4} \times \text { heartbeat mean value for } \\
\text { the whole night }\end{array}$ \\
\hline$C_{7}$ & $\begin{array}{l}\text { Respiration rate variation } \leq a_{5} \times \text { Respiration } \\
\text { rate variation mean for the whole night }\end{array}$ \\
\hline$C_{8}$ & $\begin{array}{l}\text { RR interval for every second } \geq \text { mean value of } \\
\text { RR interval for the whole night. }\end{array}$ \\
\hline
\end{tabular}

Calculation of

\section{the parameters}

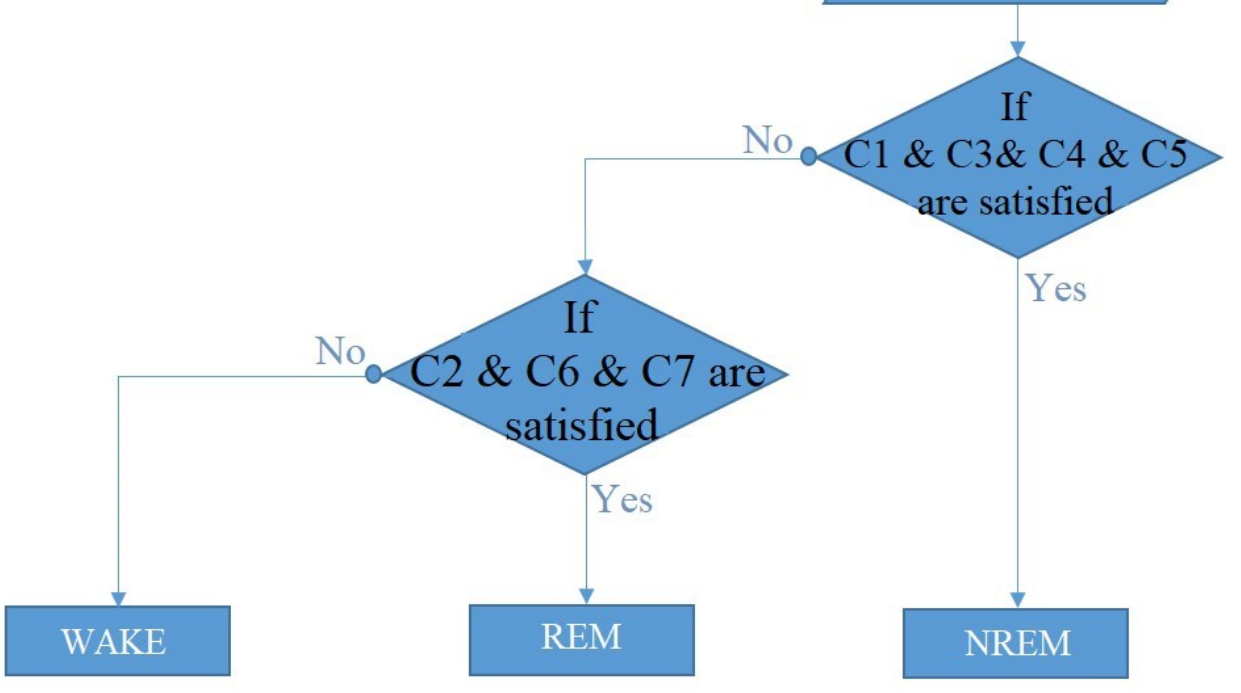

Fig. 1.Empirical classification algorithm [14] 
experiment where the sensitivity of each coefficient was tested individually (since the coefficients are independent from each other). At the end of this experiment, we came up with three different sets of values that led to three different algorithms. These algorithms were tested individually and combined [23]. The combination of the three was obtained through a voting system respecting the majority rule. In the case of a conflict, we only took the most precise of the three into consideration.

\section{B. Machine Learning Approaches}

Using the literature review, we selected five machine learning algorithms to perform the sleep stages classification: Support Vector Machine (SVM), Multinomial Logistic regression (Rlogic), k-nearest neighbours (KNN), Classification And Regression Trees (CART) and Naive Bayes (NB).

The features we used are those defined in [14]. The target is the stage detected by the PSG for each epoch. So we model the problem as a supervised classification problem with 3 classes (Wake, NREM, REM), as formally defined in Definition 4. 80\% of the collected data was used to train the machine learning algorithm and the rest was used for the validation. The classification procedure is composed of 4 steps:

1) Data processing

2) Calculation of features and storage into a CSV file

3) Training the algorithm on $80 \%$ of the data

4) Testing of the algorithm on the remaining $20 \%$ of the data

The key performance indicator is the accuracy of the algorithm, defined as Prediction 1 where tp is the number of true positive predictions and $f p$ is the number of false positive predictions.

\section{Short memory classification approach}

By doing several tests and by seeing what has been done in the past, we found that the Chattering phenomena does not characterise the sleep cycling, meaning we don't jump from one stage to another from an epoch to another. We also noticed relationships between the stages, for example it is more likely to get the "NREM" than the "REM" after a "Wake" epoch. We also noticed that a "REM" stage is more likely to happen after a "REM" stage.

From here came the idea of adding the notion of succession to the classification algorithm. From now on, the model will consider the previous value of the predicted target during the previous epoch as a new feature. So regarding the previous part of this work, the main new contribution of this article is to consider the target predicted in the previous target as a new feature, and also to take into account the movements.

To model this problem as a supervised classification problem, we added a new feature called previous state and we assume that the value of the target that comes before the starting moment of the recording is Wake $\left(t_{0}=0\right)$ since we start sleeping after being awake. We also added the previous values of the other features. Finally, each epoch is defined by features of the current epoch plus features of the previous epoch. So the new representation of an epoch $e_{i}$ is given as follows :

$$
e_{i}=\left(x_{i}-1, y_{i}-1, x_{i}, y_{i}\right)
$$

where $x_{i}$ are the values of the features at epoch $i, x_{i-1}$ are the values of the features at epoch $i-1, y_{i-1}$ is the sleep stage associated to epoch $i-1$ and $y_{i}$ is the sleep stage associated to epoch $i$.

Table 3 is an example of the new features for each epoch that we will consider, in this part of the work, as driven from the previous line. As explained earlier we consider that the first epoch has the same features values as the previous one. 
Please note that even though we have added some new features comparing to the first part of this research work, we have used the minimum possible number of features in order to fulfil our purse and have a satisfying precision and a good detection of all the stages. The positive effect of this update in the model will be proven in the next section where we are going to compare the results before and after adding the short memory notion.

TABLE IV THE

NEW FEATURE SPACE

EXAMPLE

\begin{tabular}{|c|c|c|c|c|c|c|c|c|c|c|}
\hline $\begin{array}{l}\text { Patient } \\
\text { id }\end{array}$ & $\begin{array}{l}\text { Epoch } \\
\text { id }\end{array}$ & $\begin{array}{l}\text { Heart rate of } \\
\mathrm{i}-1\end{array}$ & $\begin{array}{l}\text { RR int. i- } \\
1\end{array}$ & $\ldots$ & $\begin{array}{l}\text { Move }(0 / 1) \\
\mathrm{i}-1\end{array}$ & $\begin{array}{l}\text { stage i- } \\
1\end{array}$ & $\begin{array}{l}\text { Heart } \\
\text { rate }\end{array}$ & RR int. & ... & $\begin{array}{l}\text { Move } \\
(0 / 1)\end{array}$ \\
\hline 1 & 1 & 76 & 425 & $\ldots$ & 1 & 0 & 76 & 425 & $\ldots$ & 1 \\
\hline 1 & 2 & 76 & 425 & $\ldots$ & 1 & 0 & 76 & 425 & $\ldots$ & 0 \\
\hline$\ldots$ & $\ldots$ & $\ldots$ & $\ldots$ & $\ldots$ & $\ldots$ & $\ldots$ & $\ldots$ & $\ldots$ & $\ldots$ & $\ldots$ \\
\hline
\end{tabular}

IV. CASE STUDY

A. Data

In this article, PSG data from the clinical trial "St. Vincent's University Hospital / University College Dublin ${ }^{1 \text { " were }}$ used. This database contains 25 full overnight polysomnograms from adult subjects with suspected sleepdisordered breathing. The subjects were all aged over 18 with no heart disease or dysfunction, and they did not take any kind of drug that could alter the heart rate. From these full PSG, cardio-respiratory and body movements features were extracted to be further analysed in this study to match the data we would obtain through the use of our medical bed that only collects cardio-respiratory variables and tracks the body movements of the patient.

In particular, features related to heart signals such as the R-R interval, the heartbeat, the heart rate variability and the R-algorithm [13], features related to respiratory signals such as the respiration rate and its variations and features related to the body movement such as the leg movements. Some of these variables were not directly available in the database and were calculated based on the available features. For instance, the respiration rate was calculated based on the thorax movement. All variables were re-sampled at a $1 \mathrm{~Hz}$ sampling frequency and stored chronologically into 30 s epochs.

\section{B. Numerical results}

1) Empirical approach: The empirical method is easy to implement but performs poorly as shown in [14], with around $50 \%$ accuracy. Performance reaches $63.41 \%$ when using the proposed voting system, which remains very low.

2) Direct application of machine learning algorithms: By using five machine learning methods, we found that SVM was the most effective technique in this application with a total agreement of $76.50 \%$, compared to the classification given by the PSG. Figure 2 summarises those results.

Comparing to previous works, our method has shown very promising and satisfying results. We achieved better results than most of the algorithms that only used cardio-respiratory variables. This is mostly due to the fact that we used more subjects in our study. However [27] reaches a better precision but uses more features than what we do (78 instead of 17 here).

The main limitation of this work however, is the poor ability to detect the minor classes. Whatever was the method we always have a low precision for the minor classes comparing to the other stages. For instance, the total of REM

\footnotetext{
1 https://doi.org/10.13026/C26C7D
} 


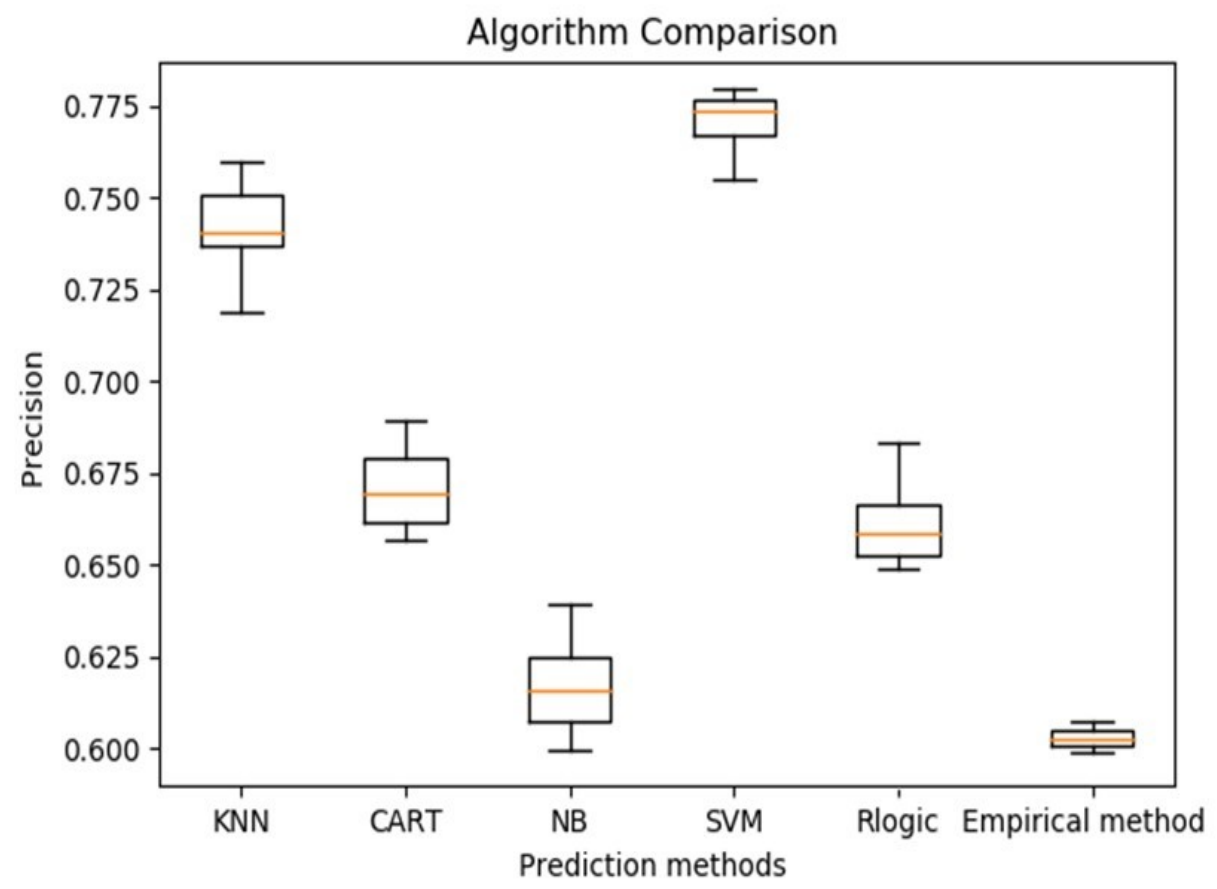

Fig. 2.Direct machine learning approach comparison

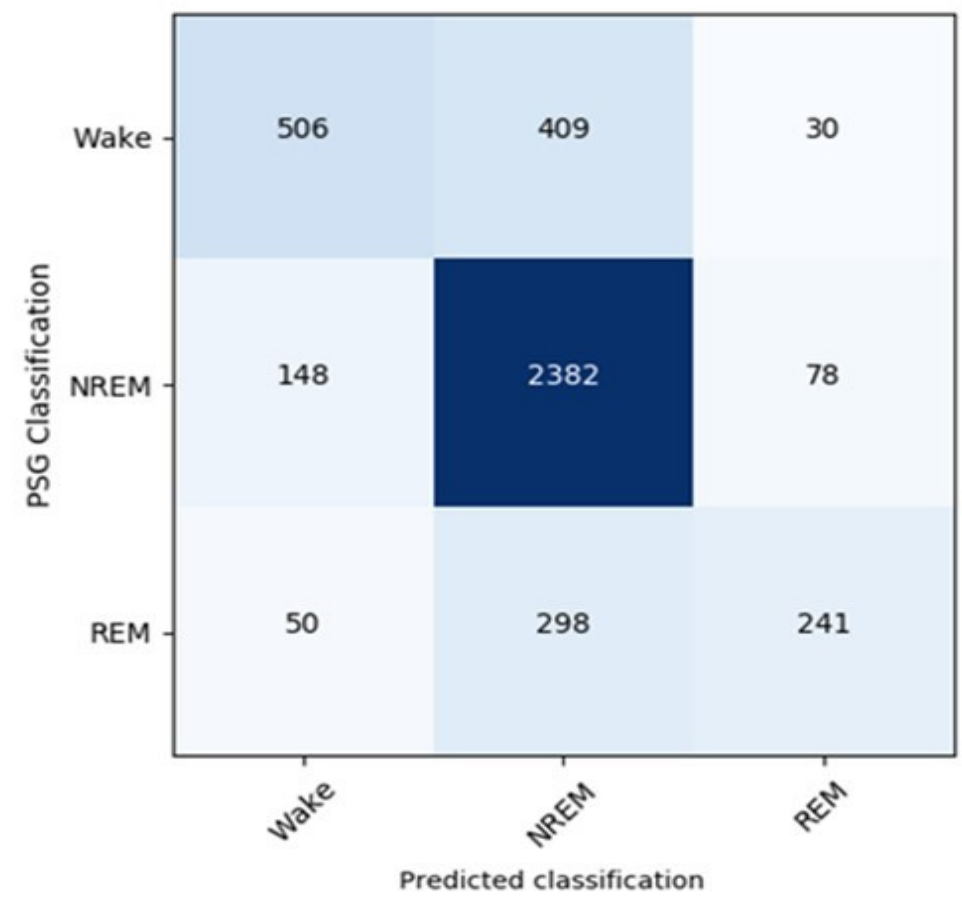

Fig. 3.Confusion Matrix for the SVM results

stage did not exceed $41 \%$ of the total agreement as shown in figure 3 . Hence came the idea of adding more information that could improve our precision.

3) Short memory classification approach: We started by testing the method that only includes the movements of the legs. We applied the usual five classical machine learning algorithms. Figure 4 presents the results obtained 
by the different algorithms. In the same way as previously discussed, we only used $80 \%$ of data to train the algorithm and left $20 \%$ to help the prediction of the stages.

As expected, adding the movement to the studied features improves the precision of the classification. We also found that SVM was the most effective algorithm since it reaches $80.32 \%$ agreement with the PSG prediction. We have then tested the effect of having a short memory (the value of the previous epoch). The same method of implementation was applied. $80 \%$ of data was used for training the algorithm and the leftover was used for the prediction of the stages. This time we conducted tests on only four

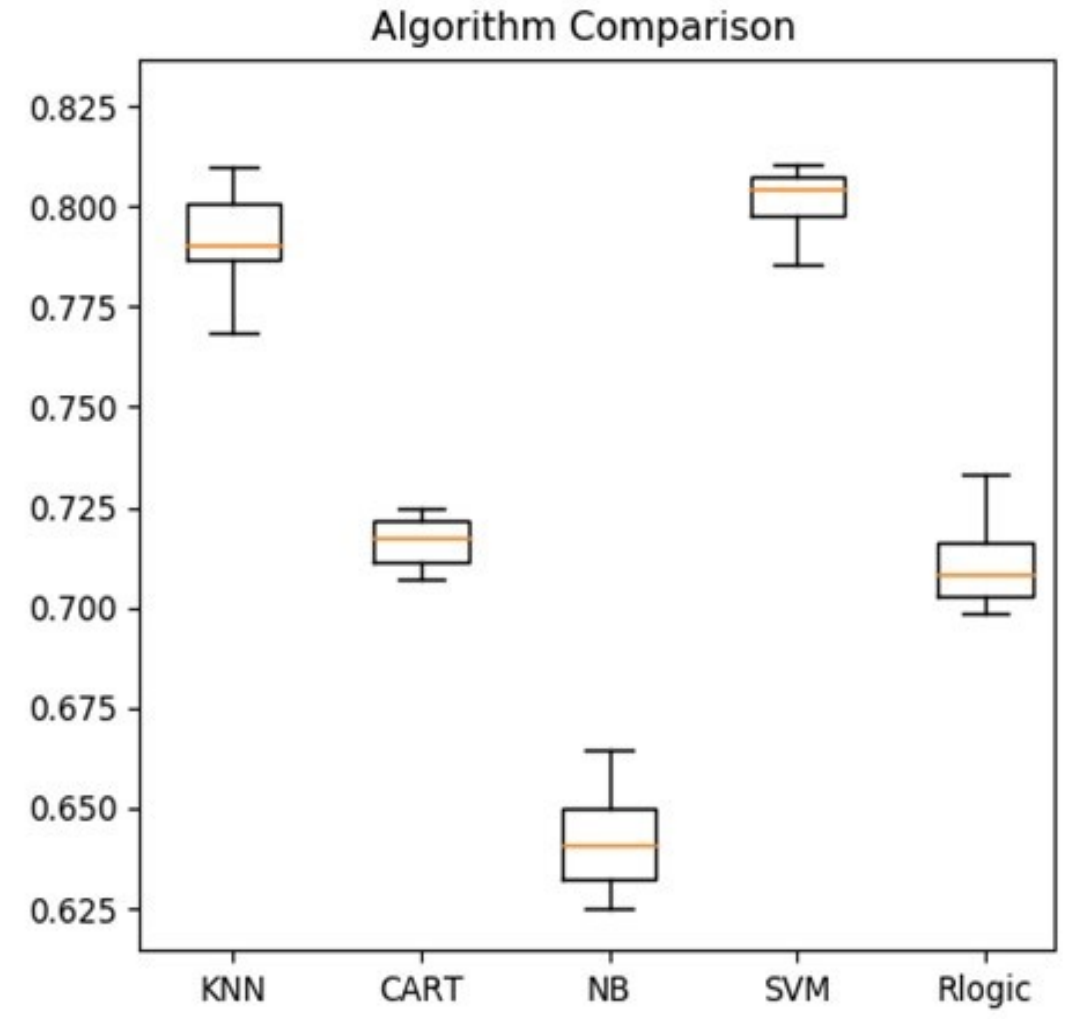

Fig. 4.A comparison of the previous used method including movement

of the algorithms: support vector machine (SVM), Linear Discriminant Analysis (LDA), Classification And Regression Trees (CART) and Neural Net (NN). 


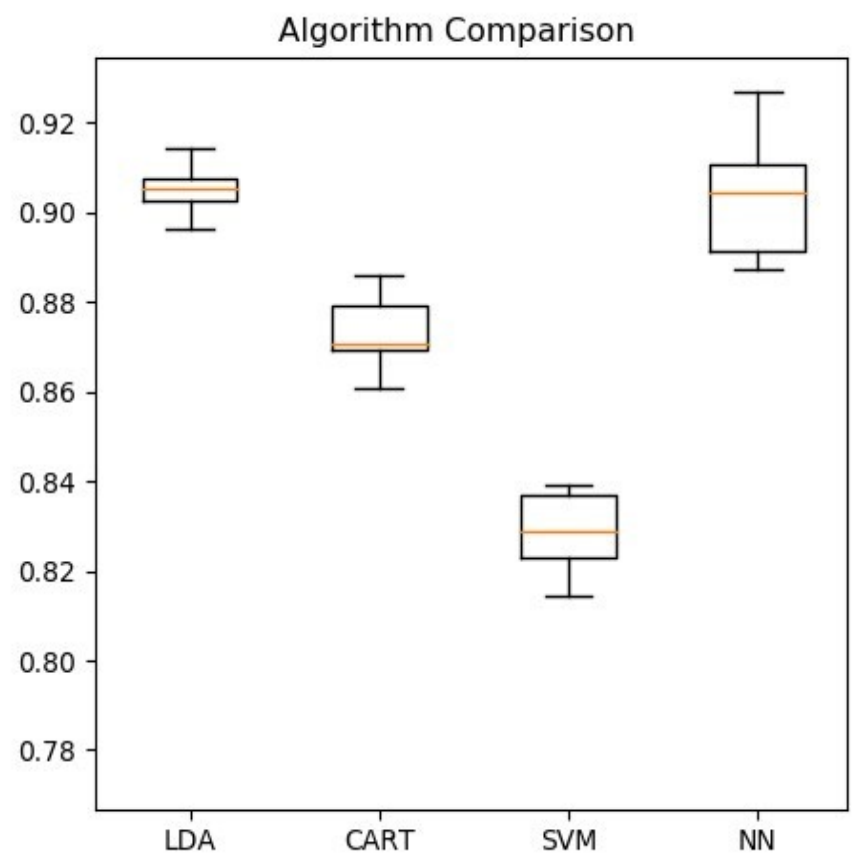

Fig. 5.A comparison of the previous used method including short memory

Using this method we found that the Neural Net (NN) was the most effective one as we reached $91.34 \%$ of agreement with the PSG as shown in figure 5.

Figure 6 shows the stages predicted by our new method vs the stages predicted from the PSG (shown as black dots on the figure). The green dots represent the correct predictions of the algorithms and the red dots represent the false predictions. This method has met our goal, since it reaches $91.34 \%$ of precision.

We can see in figure 7 that with the addition of the new two features we have improved, compared to our previous work, the detection of all the stages, even the minority classes such as REM. We found that its detection precision has reached $92 \%$.

So overall, we can say as a conclusion that we have fulfilled our goals here. We have come up with a model that uses the least features possible (19 vs 78 in [27]) and we have even a better precision in the classification and the detection of stages since we have reached over $91 \%$ agreement with the PSG. 


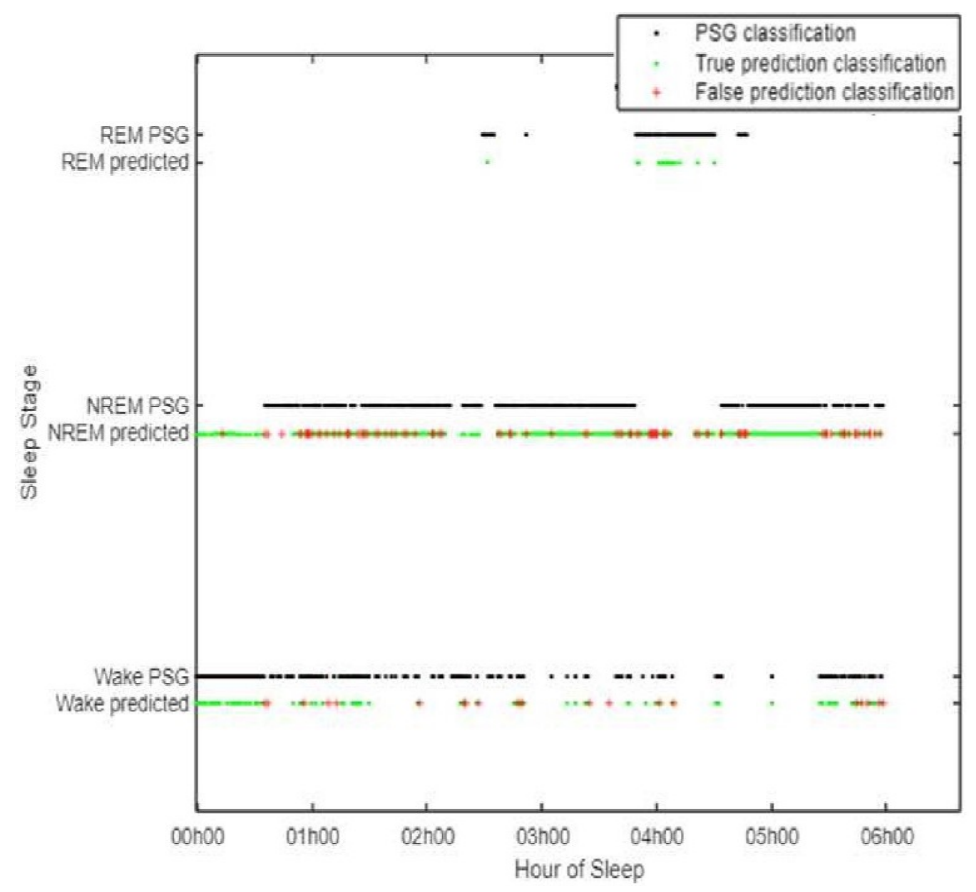

Fig. 6.Predicted night using the Neural Net (NN) with short memory

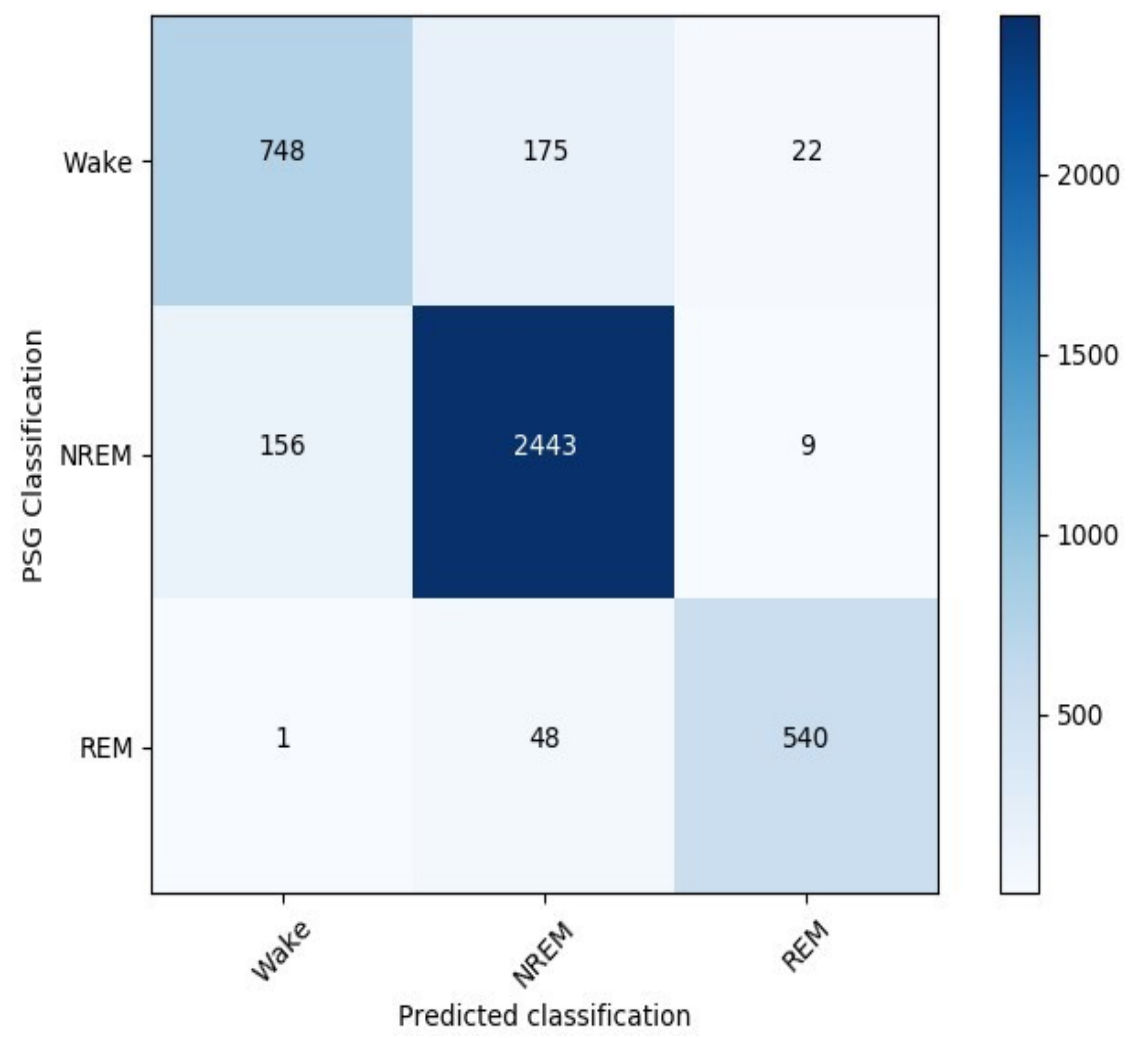

Fig. 7.Confusion Matrix for the Neural network results with short memory

\section{CONCLUSIONS AND PERSPECTIVES}

This article presents a new approach to predict sleep stage classification using a combination between cardiorespiratory variables and leg movement variables (both obtained from the new medical bed). This method was 
tested using a collected data set of 25 subjects presenting symptoms of breathing sleep apnea. Inspired from the previous publications on this topic, a choice of parameters was made.

We started by creating an empirical decision tree, based on the combination of conditions proven in previous research works. By modifying the coefficients in these conditions, we obtained three algorithms that achieved more than $50 \%$ of agreement with the results driven by the PSG using brain activities. A system of vote was designed between these three algorithm resulting in a better score agreement (up to 63.41\%).

By using five machine learning methods, we found that SVM is the most precise technique in this application with a total agreement of $76.50 \%$, compared to the classification given by the PSG. We found that, even with this satisfying precision, some difficulties in detecting the REM class remain.

To help solving this issue, we took into account the notion of leg movement. By using the same five machine learning algorithms, we reached a score of $80.32 \%$ compared to the classification given by the PSG. We have noticed a slight improvement in the REM detection so we have upgraded our model by including some knowledge of the past by memorising the last values of the features and the last value of the stage for the previous epoch. This way we achieved a $91.34 \%$ agreement with the PSG.

In our future works, we plan to conduct tests on healthier subjects: an upcoming clinical trial, whose main aim is to place a BCG sensor in a medical bed alongside with scaling sensors, should help us reach better results. This trial is part of a project aiming to link the sleep disorders with issues on cognitive impairment and deterioration in memory quality.

\section{REFERENCES}

[1] Ni, Hongbo \& Zhao, Tingzhi \& Zhou, Xingshe \& Wang, Zhu \& Chen, Lei \& Yang, Jun. Analyzing Sleep Stages in Home Environment Based on Ballistocardiography.International Conference on Health Information Science.2015. 56-68.

[2] Phan H, Andreotti F, Cooray N, Chen OY, Vos M. Automatic Sleep Stage Classification Using Single-Channel EEG: Learning Sequential Features with Attention-Based Recurrent Neural Networks. Annu Int Conf IEEE Eng Med Biol Soc. 2018 Jul;2018:1452-1455.

[3] Rechtschaffen Allan and Kales Anthony. A manual for standardized terminology, techniques and scoring system for sleep stages in human subjects. Brain Research Institute. US Dept. of Health, Education, and Welfare, 1968.

[4] A. M. Bianchi and M O Mendez. Automatic detection of sleep macrostructure based on a sensorized tshirt. 2010 Annual International Conference of the IEEE Engineering in Medicine and Biology, pages 3606-3609, 2010.

[5] Emilie Crawford Achour. Physiological and pathological sleep: impact on successful aging and cognitive function. Theses, Universite Jean Monnet - Saint-Etienne, 2013.

[6] Mary Carskadon, William Dement, Meir Kryger, Thomas Roth, and

Timothy Roehrs. Normal Human Sleep: An Overview, volume 2. 2005.

[7] Yuriko Doi, Masumi Minowa, and Toshiro Tango. Impact and Correlates of Poor Sleep Quality in Japanese White-Collar Employees. Sleep, 26(4):467-471, 2003.

[8] Kristine E. Ensrud, Terri L. Blackwell, Susan Redline, Sonia AncoliIsrael, Misti L. Paudel, Peggy M. Cawthon, Thuy-Tien L. Dam, Elizabeth BarrettConnor, Ping C. Leung, Katie L. Stone, and for the Osteoporotic Fractures in Men Study Group. Sleep disturbances and frailty status in older community-dwelling men. Journal of the American Geriatrics Society, 57(11):2085-2093, 2009.

[9] Maksym Gaiduk, Thomas Penzel, Juan Antonio Ortega, and Ralf

Seepold. Automatic sleep stages classification using respiratory, heart rate and movement signals. Physiological Measurement, page 124008, 2018.

[10] Yo-El S. Ju, Jennifer S. McLeland, Cristina D. Toedebusch, Chengjie Xiong, Anne M. Fagan, Stephen P. Duntley, John C. Morris, and David M. Holtzman. Sleep Quality and Preclinical Alzheimer DiseaseSleep Quality and Preclinical Alzheimer Disease. JAMA Neurology, 70(5):587-593, 2013.

[11] Ibrahim Sadek. Nonintrusive Nocturnal Remote Monitoring of Vital Signs in Ambient Assisted Living Environments. Systems and control, Universite Montpellier, 2018 
[12] Rechtschaffen Allan and Kales Anthony. A manual for standardized terminology, techniques and scoring system for sleep stages in human subjects. Brain Research Institute. US Dept. of Health, Education, and Welfare, 1968.

[13] Yosuke Kurihara and Kajiro Watanabe. Sleep-stage decision algorithm by using heartbeat and bodymovement signals. IEEE Transactions on Systems, Man, and Cybernetics - Part A: Systems and Humans, pages 1450-1459, 2012.

[14] Asma Gasmi, Vincent Augusto, Paul-Antoine Beaudet, Jenny Faucheu, Claire Morin, Xavier Serpaggi, Franck VASSEL, "Sleep stages classification using cardio-respiratory variables,"2020 IEEE 16th International Conference on Automation Science and Engineering (CASE), Hong Kong, Hong Kong, 2020, pp. 1031-1036*;

[15] Rao, Shashank \& El Ali, Abdallah \& Cesar, Pablo. DeepSleep: a ballistocardiographic deep learning approach for classifying sleep stages.The 2019 ACM International Joint Conference on Pervasive and Ubiquitous Computing and the 2019 ACM International Symposium.2019.187-190.

[16] Rechtschaffen Allan and Kales Anthony. A manual for standardized terminology, techniques and classification system for sleep stages in human subjects. Brain Research Institute. US Dept. of Health, Education, and Welfare, 1968.

[17] A. Rahimi, A. Safari and M. Mohebbi, "Sleep Stage Classification Based on ECG-Derived Respiration and Heart Rate Variability of

Single-Lead ECG Signal," 2019 26th National and 4th International

Iranian Conference on Biomedical Engineering (ICBME), Tehran, Iran, 2019, pp. 158-163

[18] Sun H, Ganglberger W, Panneerselvam E, Leone MJ, Quadri SA, Goparaju B, Tesh RA, Akeju O, Thomas RJ, Westover MB. Sleep staging from electrocardiography and respiration with deep learning. Sleep. 2020 Jul $13 ; 43$

[19] Zhang, Linda \& Fabbri, Daniel \& Upender, Raghu \& Kent, David. Automated Sleep Stage Scoring of the Sleep Heart Health Study Using Deep Neural Networks. Sleep. Oct 2019.21-42

[20] Mousavi S, Afghah F, Acharya UR. SleepEEGNet: Automated sleep stage scoring with sequence to sequence deep learning approach. PLoS One. 2019;14(5)

[21] Firstbeat.A Sleep Analysis Method Based on Heart Rate Variability.Firstbeat white paper.2019.7

[22] T. F. Lesmana, S. M. Isa and N. Surantha, "Sleep Stage Identification Using the Combination of ELM and PSO Based on ECG Signal and HRV," 2018 3rd International Conference on Computer and Communication Systems (ICCCS), Nagoya, 2018, pp. 258-262

[23] Rahman, Md \& Bhuiyan, Mohammed \& Hassan, Ahnaf Rashik. Sleep stage classification using single-channel EOG. Computers in Biology and Medicine. Vol 102.2018. 211-220

[24] E. R. Widasari, K. Tanno and H. Tamura, "Automatic Sleep Stage Detection Based on HRV Spectrum Analysis," 2018 IEEE International Conference on Systems, Man, and Cybernetics (SMC), Miyazaki, Japan, 2018, pp. 869874

[25] Y. Zhao, M. Li, L. Huang, D. He and H. Yang, "Sleep stage assessment based on autocorrelation and spectral analysis of heart rate variability," 2017 IEEE International Conference on Real-time Computing and Robotics (RCAR), Okinawa, 2017, pp. 448-453

[26] A. Supratak, H. Dong, C. Wu and Y. Guo, "DeepSleepNet: A Model for Automatic Sleep Stage Scoring Based on Raw Single-Channel EEG," in IEEE Transactions on Neural Systems and Rehabilitation Engineering, vol. 25, no. 11.2017.pp. 1998-2008,

[27] R. Yi, M. Enayati, J. M. Keller, M. Popescu and M. Skubic, "NonInvasive In-Home Sleep Stage Classification Using a Ballistocardiography Bed Sensor," 2019 IEEE EMBS International Conference on Biomedical \& Health Informatics (BHI), Chicago, IL, USA, 2019, pp.

1-4.

[28] A. N. Olesen, J. A. E. Christensen, H. B. D. Sorensen and P. J. Jennum, "A Noise-Assisted Data Analysis Method for Automatic

EOG-Based Sleep Stage Classification Using Ensemble Learning," 2016 38th Annual International Conference of the IEEE Engineering in Medicine and Biology Society (EMBC), Orlando, FL, 2016, pp. 3769-3772

[29] Fonseca, Pedro \& Long, Xi \& Radha, Mustafa \& Haakma, Reinder \& Aarts, R. \& Rolink, Jerome.Sleep stage classification with ECG and respiratory effort. Physiological Measurement.2015. 36(10).

[30] Levant, A.. “Chattering Analysis." IEEE Transactions on Automatic Control 55 (2010): 1380-1389. 
[31] Zhang J, Yao R, Ge W, Gao J. Orthogonal convolutional neural networks for automatic sleep stage classification based on single-channel EEG. Comput Methods Programs Biomed. 2020 Jan;183:105089. doi: 10.1016/j.cmpb.2019.105089.

[32] Ghimatgar H, Kazemi K, Helfroush MS, Pillay K, Dereymaker A, Jansen K, Vos M, Aarabi A. Neonatal EEG sleep stage classification based on deep learning and HMM. J Neural Eng. 2020 Jun 25;17(3):036031.

[33] Diykh M, Li Y, Abdulla S. EEG sleep stages identification based on weighted undirected complex networks. Comput Methods Programs Biomed. 2020 Feb;184:105116.

[34] Nakamura T, Alqurashi YD, Morrell MJ, Mandic DP. Hearables: Automatic Overnight Sleep Monitoring With Standardized In-Ear EEG Sensor. IEEE Trans Biomed Eng. 2020 Jan;67(1):203-212. 